\title{
Paeonol suppresses lipid formation and promotes lipid degradation in adipocytes
}

\author{
$\mathrm{JI} \mathrm{LI}^{1}$ and HUAN GU${ }^{2}$ \\ ${ }^{1}$ Department of Pediatrics, Guang'anmen Hospital, Chinese Academy of Traditional Chinese \\ Medicine, Beijing 100053; ${ }^{2}$ Department of Cardiology of Integrated Traditional Chinese and \\ Western Medicine, China-Japan Friendship Hospital, Beijing 100029, P.R. China
}

Received June 4, 2021; Accepted July 23, 2021

DOI: $10.3892 /$ etm.2021.11001

\begin{abstract}
Paeonol can regulate a variety of physiological and pathological processes such as thrombosis, oxidative stress, inflammation and atherosclerosis. However, its potential role and underlying mechanisms in obesity and lipid metabolism remain to be elucidated. In the present study, 3T3-L1 cells were differentiated and collected on days 4,6 and 8 . The expression levels of fatty-acid-binding protein 4 (FABP4) and microRNA (miR)-21 were detected using reverse transcription-quantitative PCR and western blot analyses. Cell viability was assessed using a Cell Counting Kit-8 assay. A miR-21 mimic was constructed and transfected into 3T3-L1 preadipocytes. Adipocyte differentiation was detected using Oil Red O staining. The proteins CD36, glucose transporter 4, peroxisome proliferator-activated receptor $\gamma($ PPAR $-\gamma)$ and adipocyte protein 2 (Ap2) were detected using western blot analysis. The expression levels of FABP4 and miR-21 were increased in differentiated 3T3-L1 cells. Paeonol exhibited no effects on cell activity, whereas it inhibited the expression levels of miR-21 in the 3T3-L1 differentiated adipocytes. Paeonol suppressed the differentiation of 3T3-L1 adipocytes and its effect was partially reversed by the overexpression of miR-21. In addition, paeonol promoted the lipid degradation of 3T3-L1 adipocytes, increased the expression levels of PPAR- $\gamma$ and Ap2, and suppressed triglyceride synthesis in these cells. These effects were partially reversed by the overexpression of miR-21. In conclusion, the findings of the present study indicated that paeonol may exert protective effects against lipid formation and promote lipid degradation in adipocytes. These data provide evidence of the regulatory effect of paeonol
\end{abstract}

Correspondence to: Dr Huan Gu, Department of Cardiology of Integrated Traditional Chinese and Western Medicine, China-Japan Friendship Hospital, 2 Sakura East Road, Chaoyang, Beijing 100029, P.R. China

E-mail: huangu00029@163.com

Key words: paeonol, lipid formation, lipid degradation, childhood obesity on adipocyte differentiation and highlight its pathological significance.

\section{Introduction}

Childhood obesity is a major public health problem worldwide (1). The prevalence of obesity among children and adolescents in China has increased from 12.3 to $37.3 \%$ between 1991 and 2015 (2). Excessive accumulation of adipose tissue, increased inflammatory response, heterotopic deposition of lipids in other tissues and elevated lipid levels in obese patients are the main factors responsible for inducing secondary diseases, such as diabetes, fatty liver, cardiovascular disease and cancer (3).

Paeonol, also known as peony, is derived from the dried bark of Paeonia suffruticosa, which belongs to Paeoniaceae (Ranunculaceae) or the dried root of $\mathrm{Xu}$ Chang qing (Asclepiadaceae) or the whole grass (4). The chemical name of paeonol is 2-hydroxy-4-methoxyacetophenone and it has a wide range of biological activities (5). For example, paeonol plays a role in numerous pharmacological actions, including antiallergic, anti-inflammatory, antitumor, cardioprotective, neuroprotective, antidiabetic and antimicrobial effects (6-8). Paeonol can regulate a variety of physiological and pathological processes, such as thrombosis, oxidative stress, inflammation and atherosclerosis (9-11). Moutan Cortex and Paeoniae Radix Rubra are common traditional Chinese medicines and reverse high-fat diet-induced metabolic disorder and restore gut microbiota homeostasis (12). However, as a major component of Moutan Cortex and Paeoniae Radix Rubra, paeonol in obesity and lipid metabolism has not been fully elucidated to the best of our knowledge.

MicroRNAs (miRNAs/miRs) are small non-coding RNA molecules of 21-23 nucleotides in length. miRNAs play an important role in the regulation of insulin secretion from pancreatic islet $\beta$ cells (13-15). Meng et al (16) demonstrated that miR-21 expression was downregulated in the endothelial progenitor cells of patients with type 2 diabetes, while Tomé-Carneiro et al (17) demonstrated that miR-21 was involved in the circulatory immune response of hypertensive drugs in type 2 diabetes. Paeonol can protect vascular endothelial cells from oxidized low-density lipoprotein-induced injury by downregulating miR-21 expression (18). The 
expression levels of miR-21 are increased during adipocyte differentiation (19). Therefore, the present study aimed to explore whether paeonol could inhibit lipid formation and promote lipid degradation in adipocytes by decreasing the expression levels of miR-21.

\section{Materials and methods}

Cell culture and transfection. 3T3-L1 preadipocytes were obtained from the American Type Culture Collection. The cells were cultured in DMEM (HyClone; Cytiva) supplemented with 10\% FBS (Gibco; Thermo Fisher Scientific, Inc.) and incubated at $37^{\circ} \mathrm{C}$ with $5 \% \mathrm{CO}_{2}$.

3T3-L1 adipocytes were grown for 2 days after contact inhibition and cultured for $48 \mathrm{~h}$ at $37^{\circ} \mathrm{C}$ with DMEM containing $0.5 \mathrm{mM} 3$-isobutyl-methylxanthine, $1 \mathrm{mM}$ dexamethasone, $10 \mathrm{mg} / \mathrm{ml}$ insulin and 10\% FBS (induction differentiation solution I). Induction differentiation solution I was discarded, and cells were cultured with DMEM containing only $10 \mathrm{mg} / \mathrm{ml}$ insulin (induction differentiation solution II) and differentiated until day 8. For paeonol treatment, paeonol (purity 99.86\%; MedChemExpress) was dissolved in DMSO (final concentration $<0.01 \%$ ). 3T3-L1 preadipocytes were treated with different concentrations (30, 60 and $120 \mu \mathrm{M})$ of paeonol for $24 \mathrm{~h}$ along with the induction differentiation solution for another $48 \mathrm{~h}$, and paeonol was renewed simultaneously with the induction differentiation solution until the cells differentiated at day 8 .

miR-21 mimic (100 $\mathrm{nM})$ and mimic negative control $(100 \mathrm{nM})$ were synthesized by Shanghai GenePharma Co., Ltd. The sequence of miR-21 mimic was 5'-UAGCUUAUC AGACUGAUGUUGA-3'. The sequence of mimic negative control was 5'-UCUGACAGUUACCAAUGCUUAA-3'. The cells were seeded into 6 -well plates at a density of $3 \times 10^{5}$ cells/well and cultured for $24 \mathrm{~h}$ at $37^{\circ} \mathrm{C}$. The aforementioned oligonucleotides were transfected into the cells using Lipofectamine ${ }^{\circledR} 2000$ (Invitrogen; Thermo Fisher Scientific, Inc.) at $37^{\circ} \mathrm{C}$ for $6 \mathrm{~h}$. Then, transfected cells were further incubated for $48 \mathrm{~h}$ at $37^{\circ} \mathrm{C}$, according to the manufacturer's protocol. The transfection efficiency was detected using reverse transcription-quantitative PCR (RT-qPCR) following $48 \mathrm{~h}$ of transfection.

Cell Counting Kit-8 (CCK-8) assay. Trypsin (Beyotime Institute of Biotechnology) was used to prepare 3T3-L1 cells in a single cell suspension. Subsequently, the cells were plated into four 96 -well plates $\left(2 \times 10^{3}\right.$ cells/well $)$ and incubated for $0,24,48$ or $72 \mathrm{~h}$. Following each incubation, CCK-8 solution (Dojindo Molecular Technologies, Inc.) was added to the culture medium in the 96-well plate and incubated for a further $1 \mathrm{~h}$. The absorbance of the cells was detected using spectrophotometry (Thermo Fisher Scientific, Inc.).

Oil Red $O$ staining. Following induction and differentiation, the cells were analyzed using Oil Red O staining. Briefly, the cells were washed with PBS solution and subsequently fixed with $4 \%$ formaldehyde for $30 \mathrm{~min}$ at room temperature. After $10 \mathrm{~min}$, a new $4 \%$ formaldehyde fixation solution was added, and the fixation was continued at room temperature for $2 \mathrm{~h}$. The fixation solution was discarded, and 60\% isopropanol solution was added to remove the residual formaldehyde solution. This was then discarded, and the cell culture plate was left in an oven at $37^{\circ} \mathrm{C}$ to dry. The prepared Oil Red $\mathrm{O}$ working solution was incubated with the cells at room temperature in the dark for 10 min. Samples were then rinsed 3-4 times with PBS to remove the residual Oil Red $\mathrm{O}$ working solution. The cell culture plate was dried in an oven at $37^{\circ} \mathrm{C}$ and a small amount of ultra-pure water was added for image capture (light microscope; Nikon Corporation). The ultrapure water was discarded, and the cell culture plates were dried in an oven at $37^{\circ} \mathrm{C}$. Isopropanol solution $(100 \%)$ was added, and the plates were placed at room temperature in the dark for $10 \mathrm{~min}$. The absorbance was measured at $490 \mathrm{~nm}$ using a microplate analyzer (MR-96A; Shenzhen Mindray Bio-Medical Electronics Co., Ltd.).

Glyceride content assay. Using the high-fat sample glycerol enzyme assay kit (cat. no. E1002; Applygen Technologies, Inc.), all procedures were performed according to the manufacturer's instructions. The cells were collected, and the lysate was added to each sample and mixed well, and incubated at room temperature for $10 \mathrm{~min}$. The upper layer of liquid from the lysate was transferred to a centrifuge tube was heated at $70^{\circ} \mathrm{C}$ for $10 \mathrm{~min}$ and centrifuged for $5 \mathrm{~min}$ at $2,795 \mathrm{x}$ g at room temperature. The supernatant obtained was used for enzymatic assays. The absorbance was measured at $490 \mathrm{~nm}$ using a microplate analyzer.

$R T-q P C R$. Total RNA was extracted from cells using TRIzol ${ }^{\circledR}$ (Invitrogen; Thermo Fisher Scientific, Inc.). Subsequently, PrimeScript $^{\mathrm{TM}}$ RT reagent Kit (Takara Bio, Inc.) was used to reverse transcribe the RNA into cDNA. The reaction conditions were as follows: $37^{\circ} \mathrm{C}$ for $15 \mathrm{~min}$ and $85^{\circ} \mathrm{C}$ for $5 \mathrm{sec}$. qPCR was subsequently performed on an ABI 7500 Real-Time PCR Detection system (Applied Biosystems; Thermo Fisher Scientific, Inc.). qPCR reactions were performed using ChamQ SYBR qPCR Master Mix (Vazyme Biotech Co., Ltd.). The thermocycling conditions were as follows: Pre-denaturation at $95^{\circ} \mathrm{C}$ for $30 \mathrm{sec}$; followed by 40 cycles of denaturation at $95^{\circ} \mathrm{C}$ for $10 \mathrm{sec}$, annealing at $55^{\circ} \mathrm{C}$ for $30 \mathrm{sec}$ and extension at $72^{\circ} \mathrm{C}$ for $1 \mathrm{~min}$. Expression levels were analyzed using the $2^{-\Delta \Delta \mathrm{Cq}}$ method (20). The primer sequences used were as follows: Fatty acid-binding protein 4 (FABP4) forward, 5'-AAGGTGAAG AGCATCATAACCCT-3' and reverse, 5'-TCACGCCTTTCA TAACACATTCC-3'; miR-21 forward, 5'-CCGCTCGAG ATCCCAGTAATGGAATGAAG-3' and reverse, 5'-ATA AGAATGCGGCCGCCATCACTTATTATTGCCTATGT-3'; peroxisome proliferator-activated receptor $\gamma$ (PPAR- $\gamma$ ) forward, 5'-GGAAGACCACTCGCATTCCTT-3' and reverse, 5'-GTAATCAGCAACCATTGGGTCA-3'; adipocyte protein 2 (Ap2) forward, 5'-CGTCCCGCACGTAGAAGAC-3' and reverse, 5'-CGCCACCGAAGAGGTTGTC-3'; U6 forward, 5'-CTCGCTTCGGCAGCACA-3' and reverse, 5'-AACGCTTCACGAATTTGCGT-3'; GAPDH forward, 5'-AGGTCGGTGTGAACGGATTTG-3' and reverse, 5'-GGG GTCGTTGATGGCAACA-3'.

Western blotting. Total protein was extracted from cells using RIPA lysis buffer (Beyotime Institute of Biotechnology). Protein concentration was determined using a BCA protein assay kit and proteins (40 $\mu \mathrm{g}$ per lane) were separated using 10\% gels via SDS-PAGE (Beyotime Institute of 
A

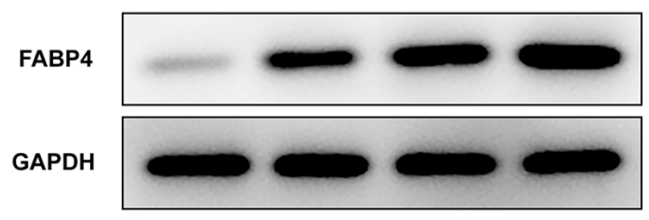

B

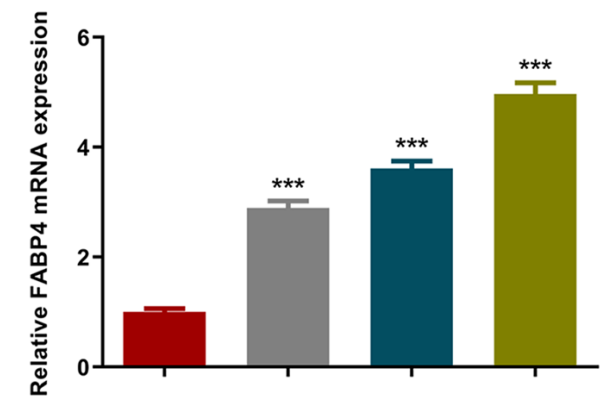

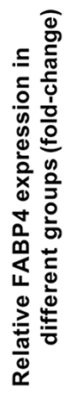

C

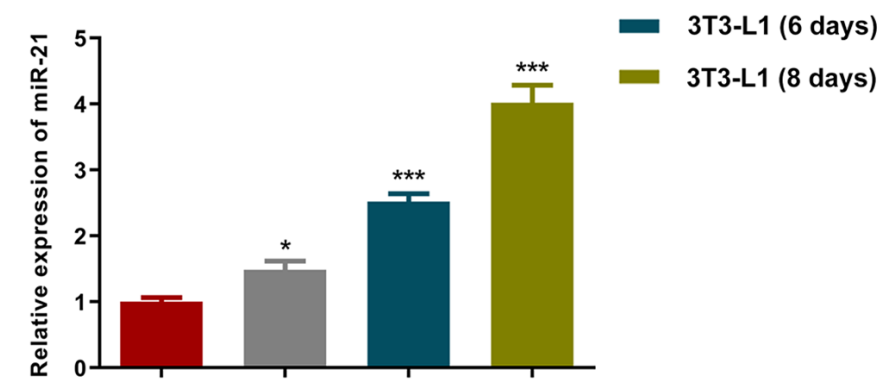

Figure 1. Expression levels of miR-21 were increased in differentiated 3T3-L1 cells. The expression levels of FABP4 were detected by (A) western blotting and (B) RT-qPCR analyses. (C) Expression levels of miR-21 were also detected using RT-qPCR analysis. "P<0.05 and ${ }^{* * *}$ P $<0.001$ vs. 3T3-L1 (undifferentiated cells). miR, microRNA; FABP4, fatty-acid-binding protein 4; RT-qPCR, reverse transcription-quantitative PCR.

Biotechnology). Following electrophoresis, the proteins were transferred to polyvinylidene fluoride membranes (MilliporeSigma) and blocked with $5 \%$ skimmed milk powder for $2 \mathrm{~h}$ at room temperature (diluted in PBS-0.1\% Tween-20). The membranes were subsequently incubated overnight at $4^{\circ} \mathrm{C}$ with the following primary antibodies: Anti-FABP4 (1:1,000; cat. no. ab92501; Abcam), anti-CD36 (1:1,000; cat. no. ab221605; Abcam), anti-glucose transporter 4 (GLUT4; 1:1,000; cat. no. ab216661; Abcam), anti-PPAR- $\gamma$ (1:1,000; cat. no. ab272718; Abcam), anti-Ap2 (1:1,000; cat. no. ab108311; Abcam) and anti-GAPDH (1:1,000; cat. no. ab8245; Abcam). Following the primary antibody incubation, the membranes were washed with PBS-0.1\% Tween-20 three times and incubated with goat anti-mouse IgG H\&L (HRP) $(1: 2,000$; cat. no. ab6789; Abcam) or goat anti-rabbit IgG H\&L (HRP) (1:3,000; cat. no. ab6721; Abcam) at room temperature for $2 \mathrm{~h}$. All antibodies were diluted in PBS-0.1\% Tween-20. Protein bands were visualized using Pierce Western Blotting substrate (Thermo Fisher Scientific, Inc.) and densitometric analysis was performed using ImageJ software (version 1.48v; National Institutes of Health).

Statistical analysis. All experiments were independently repeated at least three times and the data are presented as the mean \pm SD. Statistical analyses were performed using SPSS 19.0 software (IBM Corp.). One-way ANOVA followed by Tukey's post hoc test was used to evaluate statistical differences between groups in all experiments. $\mathrm{P}<0.05$ was considered to indicate a statistically significant difference.

\section{Results}

Expression levels of $m i R-21$ are increased in 3T3-L1 cells following differentiation. FABP4 is one of the markers of adipocyte differentiation (21). The expression levels of FABP4 were detected using RT-qPCR and western blot analyses. The increase in cell differentiation time resulted in gradual upregulation in the expression levels of FABP4 (Fig. 1A and B). The results of the RT-qPCR analysis indicated that the expression levels of miR-21 were also increased during adipocyte differentiation (Fig. 1C).

Paeonol inhibits the expression levels of miR-21 in 3T3-L1 differentiated adipocytes. To investigate the effects of paeonol on adipocytes, different concentrations of this compound were added to the differentiation medium. A CCK-8 assay was used to detect cell viability and the results revealed that the different concentrations of paeonol exhibited no effects on cell viability (Fig. 2A). However, the RT-qPCR results indicated that the expression levels of $\mathrm{miR}-21$ were decreased following an increase in paeonol concentration after the addition of differentiation medium to the cells (Fig. 2B). Therefore, the $120 \mu \mathrm{M}$ paeonol group, which exhibited the highest inhibitory effect, was selected for use in subsequent experiments.

Paeonol inhibits the adipocyte differentiation of 3T3-L1 cells, while the overexpression of miR-21 reverses this effect. To further explore the specific mechanism of paeonol-mediated inhibition of 3T3-L1 adipocyte differentiation, miR-21 mimics were transfected into the cells and the expression levels of miR-21 were assessed using RT-qPCR. The results indicated that the expression levels of miR-21 were significantly upregulated in the miR-21 mimic group (Fig. 3A). The differentiation of adipocytes was detected by Oil Red $\mathrm{O}$ staining (Fig. 3B). On the eighth day of differentiation, lipid accumulation was present in the cells and the staining was positive. Following addition of paeonol, the number of positively stained cells was significantly decreased. Following transfection with the miR-21 mimic, however, the number 
A

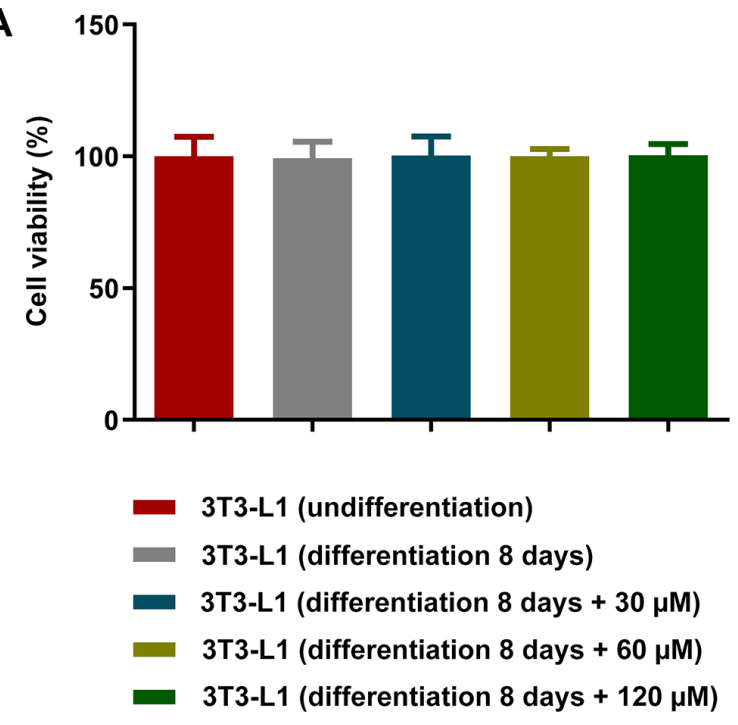

B

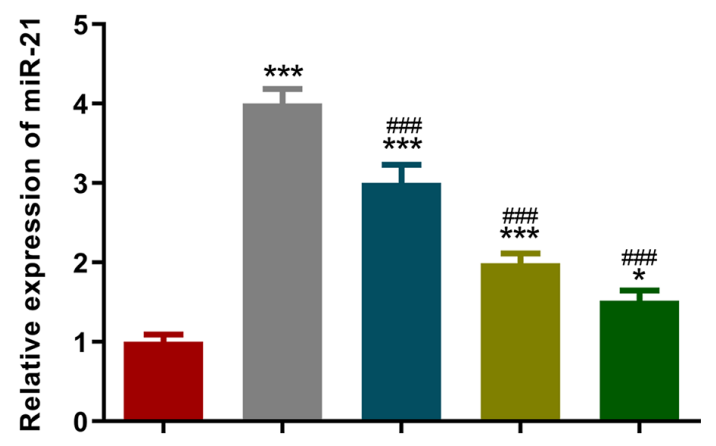

Figure 2. Paeonol inhibits the expression levels of miR-21 in 3T3-L1 differentiated adipocytes. (A) Cell Counting Kit-8 assay was used to detect cell viability. (B) Expression levels of miR-21 were detected using reverse transcription-quantitative PCR. " $\mathrm{P}<0.05$ and ${ }^{* * * *} \mathrm{P}<0.001$ vs. 3T3-L1 (undifferentiated cells);

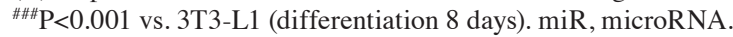

A

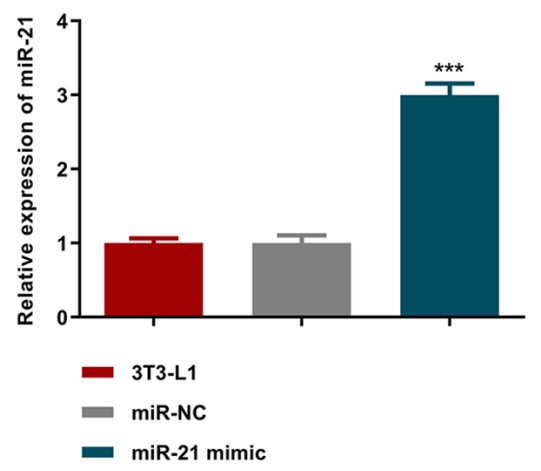

C

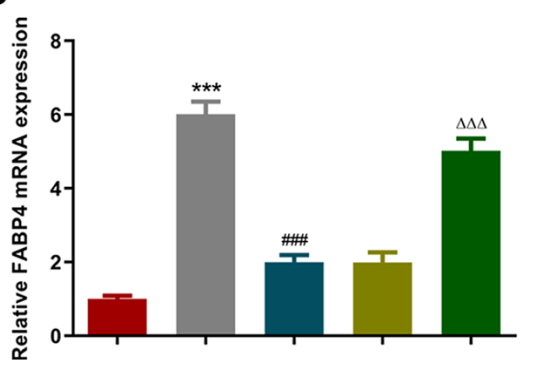

B

3T3-L1 (undifferentiation) 3T3-L1 (differentiation 8 days)
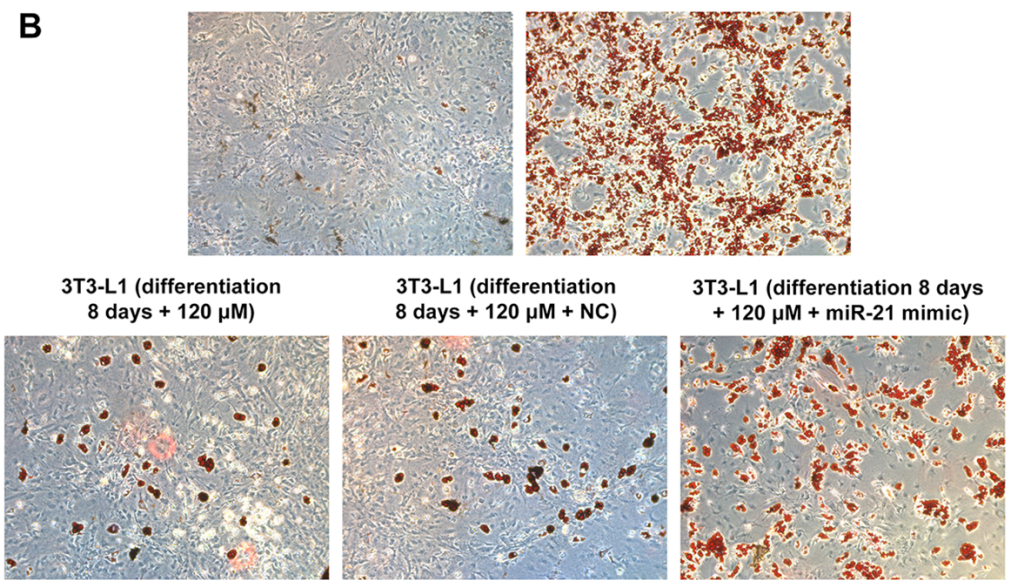

D
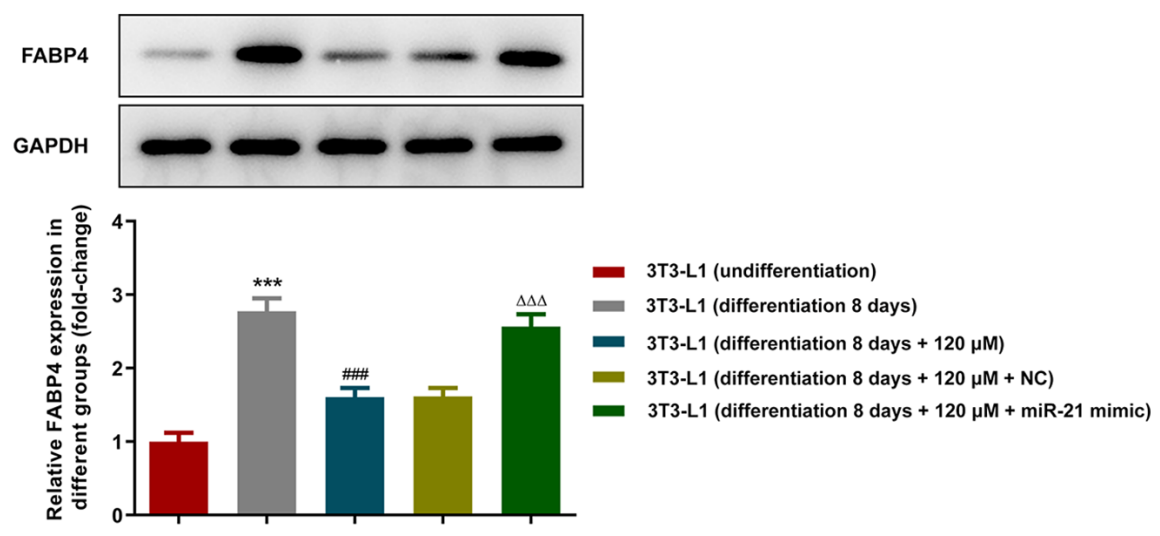

Figure 3. Paeonol inhibits adipocyte differentiation of 3T3-L1 cells and overexpression of miR-21 reverses this effect. (A) Expression levels of miR-21 were detected using RT-qPCR. (B) Differentiation of adipocytes was detected using Oil Red O staining (magnification, x200). The expression levels of the adipocyte differentiation marker, FABP4, were detected using (C) RT-qPCR and (D) western blotting analyses. ${ }^{* * *} \mathrm{P}<0.001$ vs. 3T3-L1 or 3T3-L1 (undifferentiated cells); ${ }^{\# \# \# ~ P<0.001 ~ v s . ~ 3 T 3-L 1 ~(d i f f e r e n t i a t i o n ~} 8$ days); ${ }^{\Delta \Delta \Delta} \mathrm{P}<0.001$ vs. 3T3-L1 (differentiation 8 days $+120 \mu \mathrm{M}+\mathrm{NC}$ ). miR, microRNA; RT-qPCR, reverse transcription-quantitative PCR; FABP4, fatty-acid-binding protein 4; NC, negative control.

of adipocytes stained with Oil Red O was significantly increased. Then, as demonstrated by RT-qPCR as well as western blotting results (Fig. 3C and D), paeonol inhibited the expression levels of mRNA as well as protein of the 
A

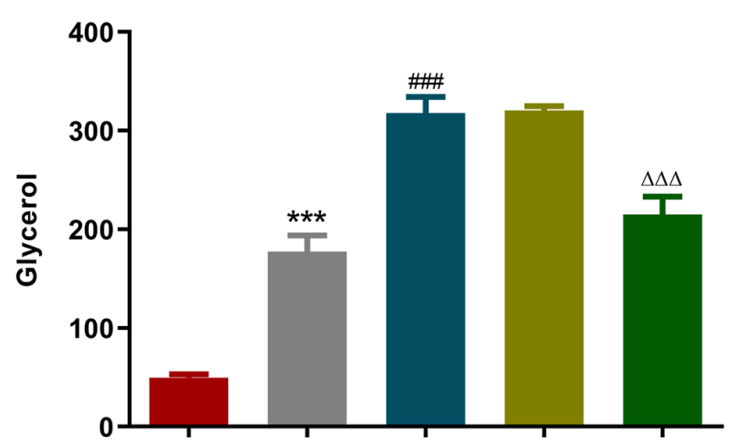

C

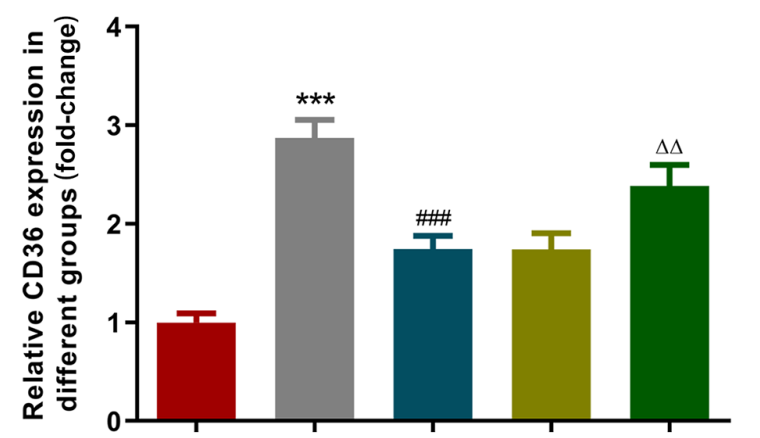

3T3-L1 (undifferentiation)

3T3-L1 (differentiation 8 days)

3T3-L1 (differentiation 8 days $+120 \mu \mathrm{M}$ )

3T3-L1 (differentiation 8 days $+120 \mu \mathrm{M}+\mathrm{NC}$ )

3T3-L1 (differentiation 8 days $+120 \mu \mathrm{M}+\mathrm{miR}-21$ mimic)
B
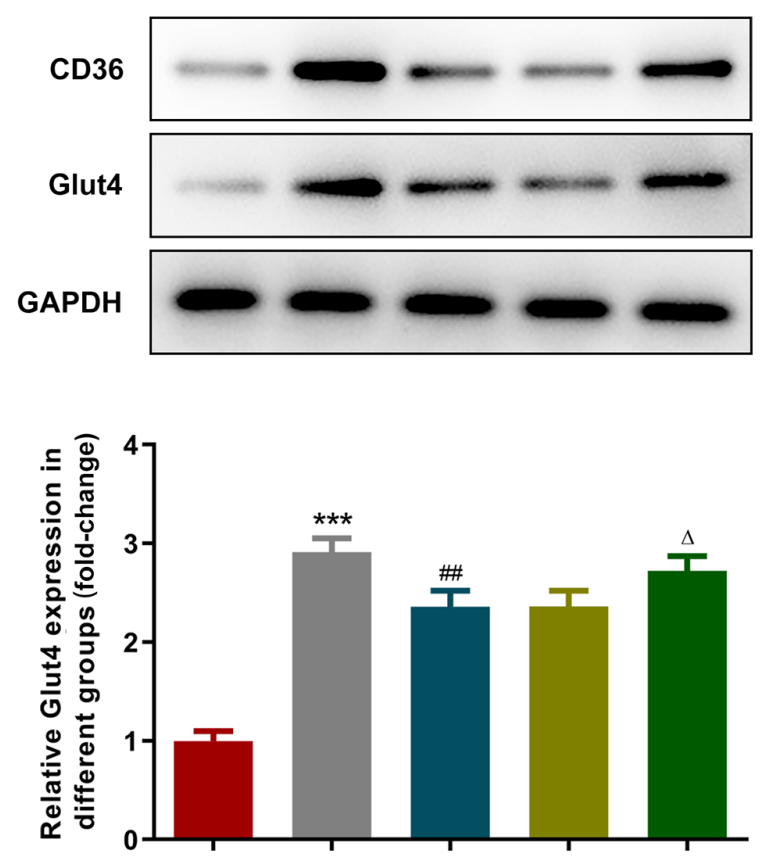

Figure 4. Paeonol promotes lipid degradation and inhibits TG synthesis in adipocytes, while overexpression of miR-21 reverses these effects. (A) GPO-Trinder was used to measure glycerin production in the supernatant. (B) Western blotting and (C) RT-qPCR analysis were used to detect the expression levels of the inflammatory protein CD36 and the synthesis of GLUT4 by TGs. ${ }^{* * * *} \mathrm{P}<0.001$ vs. 3T3-L1 (undifferentiated cells); ${ }^{\# \#} \mathrm{P}<0.01$ and ${ }^{\# \# \# P<0.001 ~ v s . ~ 3 T 3-L 1 ~(d i f f e r e n t i a t i o n ~} 8$ days); ${ }^{\Delta} \mathrm{P}<0.05,{ }^{\Delta \Delta} \mathrm{P}<0.01$ and ${ }^{\Delta \Delta \Delta} \mathrm{P}<0.001$ vs. 3T3-L1 (differentiation 8 days $+120 \mu \mathrm{M}+\mathrm{NC}$ ). TG, triglyceride; miR, microRNA; GLUT4, glucose transporter 4 .

adipocyte differentiation marker FABP4, while miR-21 mimic could partially reverse the effect of paeonol on FABP4 expression inhibition.

Paeonol promotes lipid degradation and inhibits triglyceride (TG) synthesis and the expression of adipogenic transcription factors in adipocytes, whereas overexpression of $\mathrm{miR}-21$ reverses these effects. High-fat sample glycerol enzyme assay kit was used to measure glycerol production in the supernatant (Fig. 4A). Glycerol levels were increased following the differentiation of 3T3-L1 cells. Paeonol promoted the glycerol degradation of adipocytes, while the overexpression of miR-21 reversed its effect. Western blot analysis was used to detect the expression levels of the TG metabolism-associated proteins CD36 and GLUT4. Paeonol inhibited TG synthesis, while the overexpression of miR-21 reversed this effect (Fig. 4B and C). Furthermore, the expression levels of the adipogenic transcription factors, PPAR $\gamma$ and AP2, were detected using RT-qPCR (Fig. 5A) and western blot (Fig. 5B) analyses. The expression levels of PPAR $\gamma$ and AP2 were increased in 3T3-L1 cells following cell differentiation, whereas they were decreased following treatment with paeonol. Overexpression of miR-21 also reversed the changes in expression level of PPAR $\gamma$ and AP2.

\section{Discussion}

Adipocytes originate from mesenchymal stem cells (MSCs) (22). MSCs form preadipocytes, which gradually accumulate fat droplets and differentiate into mature adipocytes following growth inhibition, clonal proliferation and gene regulation (23). Previous studies have shown that the volume of adipocytes cannot be increased infinitely. When the volume of adipocytes exceeds 1.2-1.6 $\mu \mathrm{g}$ adipocytes/cells, preadipocytes are activated via paracrine mechanisms. This in turn induces their proliferation and differentiation into adipocytes (24). Therefore, the proliferation and differentiation of preadipocytes leads to the rapid proliferation of mature adipocytes, which is one of the mechanisms responsible for causing obesity. In a previous study, 3T3-L1 preadipocytes were cloned and amplified from Swiss3T3 mouse embryonic fibroblasts. Following induction in vitro, 3T3-L1 preadipocytes differentiated into mature adipocytes, which can be used as an in vitro model to simulate the adipocyte differentiation process and the function of viable adipocyte tissue (25). 3T3-L1 preadipocytes are internationally recognized cell lines for the study of the adipogenic process (26). Therefore, this study used 3T3-L1 cells as the basis to explore the role of paeonol. 
A

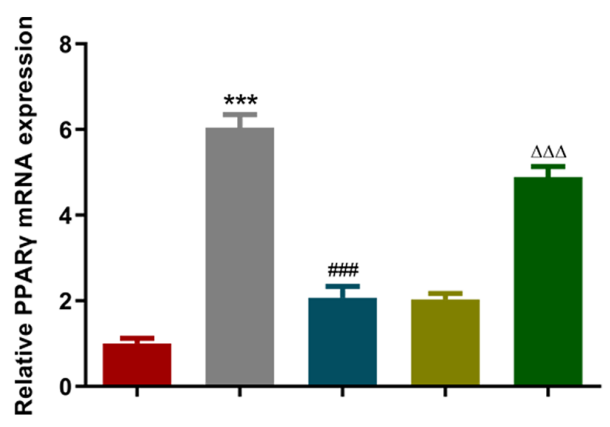

B
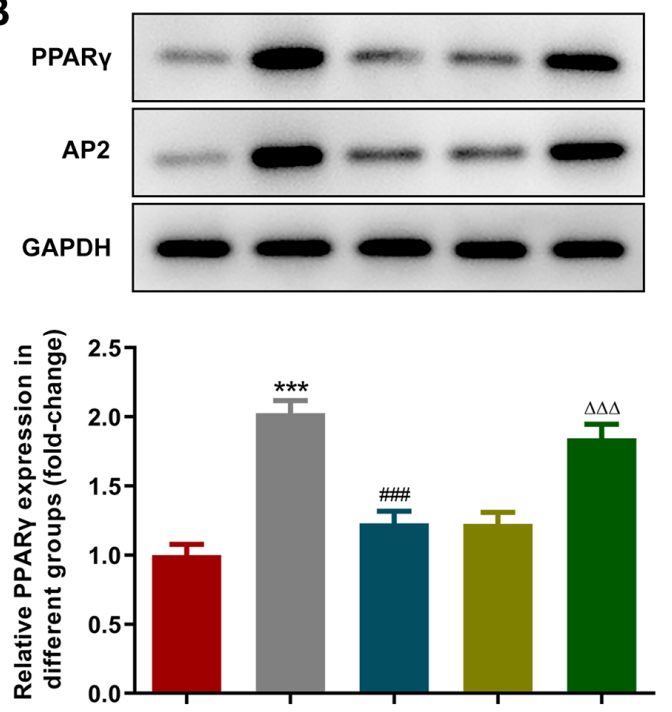
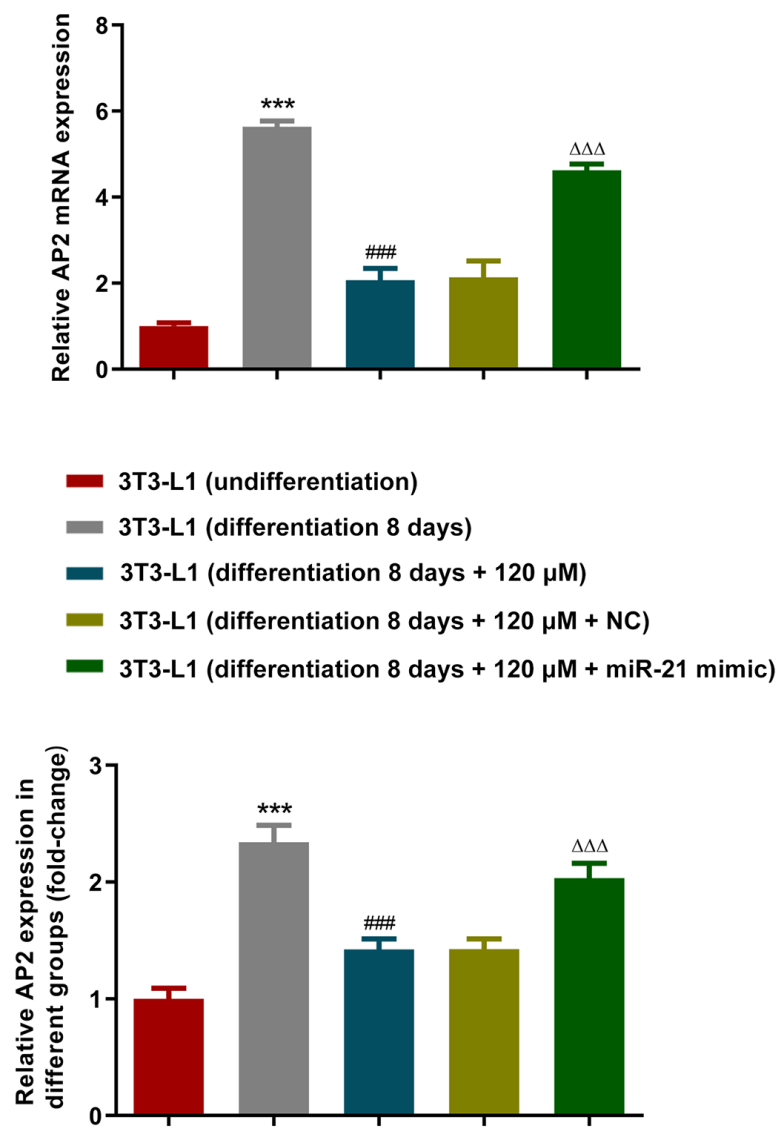

Figure 5. Paeonol inhibits the induction of adipogenic transcription factor expression in adipocytes, while overexpression of miR-21 reverses these effects. The expression levels of the adipogenic transcription factors PPAR $\gamma$ and Ap2 were detected using (A) reverse transcription-quantitative PCR and (B) western blotting analyses. ${ }^{* * *} \mathrm{P}<0.001$ vs. 3T3-L1 (undifferentiated cells); ${ }^{\# \# \# ~} \mathrm{P}<0.001$ vs. 3T3-L1 (differentiation 8 days); ${ }^{\Delta \Delta \Delta} \mathrm{P}<0.001$ vs. 3T3-L1 (differentiation 8 days $+120 \mu \mathrm{M}+\mathrm{NC})$. miR, microRNA; PPAR $\gamma$, peroxisome proliferator-activated receptor $\gamma$; Ap2, adipocyte protein 2 .

The anti-inflammatory and antioxidant effects of paeonol have been noted in specific inflammatory diseases, such as periodontitis and dermatitis (27). Paeonol significantly inhibits periodontitis-associated inflammation and oxidative stress levels by regulating the nuclear factor erythroid-2/NF- $\kappa B$ signaling pathway $(27,28)$. In a previous study, an emollient that contained paeonol was found to significantly improve the skin moisture content, $\mathrm{pH}$ and water shunt loss rate of a mouse atopic dermatitis model, without causing skin irritation (29). These aforementioned studies demonstrated the anti-inflammatory and antioxidant effects of paeonol. In the present study, the role of paeonol in lipids associated with obesity was further investigated. The experimental results of the current study demonstrated that paeonol promoted lipid degradation in adipoblast cells, inhibited TG synthesis and lipid formation in adipoblast cells and promoted lipid degradation.

The differentiation of adipocytes is a complex process regulated by transcription factors (30). Members of the PPAR family are major transcription factors that regulate adipocyte differentiation. A previous study reported that anthocyanin extract could significantly decrease the expression levels of the adipocyte differentiation-related transcription factors, PPAR $\gamma$ and Ap2 (31). The results of the present study showed that paeonol inhibited the expression of adipogenic transcription factors in 3T3-L1 adipocytes, and overexpression of miR-21 reversed its effect. The accumulation of TGs in adipocytes can reflect the degree of differentiation (32). Oil Red O can bind to neutral lipids (33). In the present study, the data indicated that paeonol inhibited the adipocyte differentiation of 3T3-L1 cells. Oil Red O staining analysis indicated that paeonol promoted lipid degradation and inhibited TG synthesis in adipoblast cells, while the overexpression of miR-21 reversed this effect.

In conclusion, the current study investigated the effects of paeonol on the proliferation and differentiation of 3T3-L1 preadipocytes and discussed its effects on lipid formation and promote lipid degradation in adipocytes. However, due to the lack of time and funding for this study, research on miR-21 and its target genes were limited and require further elucidation. Previous studies have shown that miR-21 regulates the proliferation and apoptosis of ovarian cancer cells through PTEN/PI3K/Akt, and bioinformatics analysis has found that there are complementary binding sites between miR-21 and the 3'-untranslated region of PTEN (34). Therefore, future studies will further investigate the effects of miRNAs associated with paeonol and their target genes on the biological functions of obesity and lipid metabolism by establishing animal and cell models.

\section{Acknowledgements}

Not applicable. 


\section{Funding}

The present study received a grant from the China Center for Evidence Based Traditional Chinese Medicine (no. zz13-024-5).

\section{Availability of data and materials}

The datasets used and/or analyzed during the current study are available from the corresponding author on reasonable request.

\section{Authors' contributions}

JL and HG designed the experimental study. JL analyzed the experiment data and wrote the manuscript. HG aided in correcting the manuscript. JL carried out the experiments. $\mathrm{JL}$ and HG confirm the authenticity of all the raw data. Both authors have read and approved the final manuscript.

\section{Ethics approval and consent to participate}

Not applicable.

\section{Patient consent for publication}

Not applicable.

\section{Competing interests}

The authors declare that they have no competing interests.

\section{References}

1. Masukume G, O'Neill SM, Baker PN, Kenny LC, Morton SMB and Khashan AS: The impact of Caesarean section on the risk of childhood overweight and obesity: new evidence from a contemporary cohort study. Sci Rep 8: 15113, 2018.

2. Wang L, Wang H, Zhang B, Popkin BM and Du S: Elevated fat intake increases body weight and the risk of overweight and obesity among Chinese adults: 1991-2015 trends. Nutrients 12: 3272,2020

3. Akici N, Onal ZE, Gürbüz T, Sağ C and Kilinç S: Atherogenic indices in the assessment of cardiovascular disease risk in children with obesity and subclinical hypothyroidism. Acta Endocrinol (Bucur) 16: 334-338, 2020.

4. Izumi M, Yoshida T, Nakamura T and Wakamori AM: Paeonol, an Ingredient of Kamishoyosan, Reduces Intracellular Lipid Accumulation by Inhibiting Glucocorticoid Receptor Activity in 3T3-L1 Cells. Nutrients 12: 12, 2020.

5. Al-Taher AY, Morsy MA, Rifaai RA, Zenhom NM and Abdel-Gaber SA: Paeonol attenuates methotrexate-induced cardiac toxicity in rats by inhibiting oxidative stress and suppressing TLR4-induced NF- $\mathrm{BB}$ inflammatory pathway. Mediators Inflamm 2020: 8641026, 2020.

6. Ramachandhiran D, Vinothkumar V and Babukumar S: Paeonol exhibits anti-tumor effects by apoptotic and anti-inflammatory activities in 7,12-dimethylbenz(a)anthracene induced oral carcinogenesis. Biotech Histochem 94: 10-25, 2019.

7. Adki KM and Kulkarni YA: Neuroprotective effect of paeonol in streptozotocin-induced diabetes in rats. Life Sci 271: 119202, 2021.

8. Xu F, Xiao H, Liu R, Yang Y, Zhang M, Chen L, Chen Z, Liu P and Huang H: Paeonol ameliorates glucose and lipid metabolism in experimental diabetes by activating Akt. Front Pharmacol 10 : 261, 2019.

9. Ping M, Xiao W, Mo L, Xiao X, Song S, Tang W and Yang X: Paeonol attenuates advanced oxidation protein product-induced oxidative stress injury in THP-1 macrophages. Pharmacology 93: 286-295, 2014
10. Li H, Dai M and Jia W: Paeonol attenuates high-fat-diet-induced atherosclerosis in rabbits by anti-inflammatory activity. Planta Med 75: 7-11, 2009.

11. Yu Y, Yan R, Chen X, Sun T and Yan J: Paeonol suppresses the effect of ox-LDL on mice vascular endothelial cells by regulating miR-338-3p/TET2 axis in atherosclerosis. Mol Cell Biochem 475: 127-135, 2020.

12. Zhong LJ, Xie ZS, Yang H, Li P and Xu XJ: Moutan Cortex and Paeoniae Radix Rubra reverse high-fat-diet-induced metabolic disorder and restore gut microbiota homeostasis. Chin J Nat Med 15: 210-219, 2017.

13. Melkman-Zehavi T, Oren R, Kredo-Russo S, Shapira T, Mandelbaum AD, Rivkin N, Nir T, Lennox KA, Behlke MA, Dor Y, et al: miRNAs control insulin content in pancreatic $\beta$-cells via downregulation of transcriptional repressors. EMBO J 30: 835-845, 2011.

14. MaE, Fu Y and Garvey WT: Relationship of circulating miRNAs with insulin sensitivity and associated metabolic risk factors in humans. Metab Syndr Relat Disord 16: 82-89, 2018.

15. Lang H, Xiang Y, Lin N, Ai Z, You Z, Xiao J, Liu D and Yang Y: Identification of a panel of miRNAs as positive regulators of insulin release in pancreatic $\beta$-cells. Cell Physiol Biochem 48: 185-193, 2018.

16. Meng S, Cao JT, Zhang B, Zhou Q, Shen CX and Wang CQ: Downregulation of microRNA-126 in endothelial progenitor cells from diabetes patients, impairs their functional properties, via target gene Spred-1. J Mol Cell Cardiol 53: 64-72, 2012.

17. Tomé-Carneiro J, Larrosa M, Yáñez-Gascón MJ, Dávalos A, Gil-Zamorano J, Gonzálvez M, García-Almagro FJ, Ruiz Ros JA, Tomás-Barberán FA, Espín JC, et al: One-year supplementation with a grape extract containing resveratrol modulates inflammatory-related microRNAs and cytokines expression in peripheral blood mononuclear cells of type 2 diabetes and hypertensive patients with coronary artery disease. Pharmacol Res 72: 69-82, 2013

18. Liu YR, Chen JJ and Dai M: Paeonol protects rat vascular endothelial cells from ox-LDL-induced injury in vitro via downregulating microRNA-21 expression and TNF- $\alpha$ release. Acta Pharmacol Sin 35: 483-488, 2014.

19. Kang M, Yan LM, Zhang WY, Li YM, Tang AZ and Ou HS: Role of microRNA-21 in regulating 3T3-L1 adipocyte differentiation and adiponectin expression. Mol Biol Rep 40: 5027-5034, 2013.

20. Livak KJ and Schmittgen TD: Analysis of relative gene expression data using real-time quantitative PCR and the 2(-Delta Delta C(T)) method. Methods 25: 402-408, 2001.

21. Mitterberger MC, Lechner S, Mattesich M, Kaiser A, Probst D, Wenger N, Pierer $G$ and Zwerschke W: DLK1(PREF1) is a negative regulator of adipogenesis in $\mathrm{CD} 105^{+} / \mathrm{CD} 0^{+} / \mathrm{CD} 34^{+} / \mathrm{CD} 31^{-} / \mathrm{FABP} 4-$ adipose-derived stromal cells from subcutaneous abdominal fat pats of adult women. Stem Cell Res 9: 35-48, 2012

22. Lee SC, Lee YJ, Shin MK and Sung JS: Regulation of CXCR6 expression on adipocytes and osteoblasts differentiated from human adipose tissue-derived mesenchymal stem cells. Stem Cells Int 2020: 8870133, 2020.

23. Jiang R, Yang T, Zhang Y, Wang Z and Zhang T: LKB1 promotes the transformation of bone marrow mesenchymal stem cells into adipocytes under oxidative stress via AMPK-mTOR signaling pathway. J Interferon Cytokine Res 40: 370-376, 2020.

24. Zhang J, Cai B, Ma M, Luo W, Zhang Z, Zhang X and Nie Q: ALDH1A1 inhibits chicken preadipocytes' proliferation and differentiation via the PPAR $\gamma$ pathway in vitro and in vivo. Int $\mathbf{J}$ Mol Sci 21: 3150, 2020.

25. Pacifici F, Farias CLA, Rea S, Capuani B, Feraco A, Coppola A, Mammi C, Pastore D, Abete P, Rovella V, et al: Tyrosol may prevent obesity by inhibiting adipogenesis in 3T3-L1 preadipocytes. Oxid Med Cell Longev 2020: 4794780, 2020.

26. Son Y, Cox JM, Stevenson JL, Cooper JA and Paton CM: Angiopoietin-1 protects 3T3-L1 preadipocytes from saturated fatty acid-induced cell death. Nutr Res 76: 20-28, 2020.

27. Kurt S, Gürkan CG, Keles Tezal GC, Ciftçi A, Gürgör PN, Güler Ş and Çetinkaya BÖ: Histopathological and biochemical evaluation of the effect of Paeoniflorin on the periodontium during and after periodontitis formation in rats. Arch Oral Biol 102: 135-140, 2019.

28. Ni J, Yang D, Song L and Li C: Protective effects of paeoniflorin on alveolar bone resorption and soft-tissue breakdown in experimental periodontitis. J Periodontal Res 51: 257-264, 2016. 
29. Qiu J, Chen M, Liu J, Huang X, Chen J, Zhou L, Ma J, Sextius P, Pena AM, Cai Z, et al: The skin-depigmenting potential of Paeonia lactiflora root extract and paeoniflorin: In vitro evaluation using reconstructed pigmented human epidermis. Int J Cosmet Sci 38: 444-451, 2016.

30. Baek K and Baek JH: The transcription factors myeloid elf-1-like factor (MEF) and distal-less homeobox 5 (Dlx5) inversely regulate the differentiation of osteoblasts and adipocytes in bone marrow. Adipocyte 2: 50-54, 2013.

31. Wang P, Ji R, Ji J and Chen F: Changes of metabolites of acrylamide and glycidamide in acrylamide-exposed rats pretreated with blueberry anthocyanins extract. Food Chem 274: 611-619, 2019.
32. Kim JH, Lee S, Kim HY and Cho EJ: Acer okamotoanum inhibits adipocyte differentiation by the regulation of adipogenesis and lipolysis in 3T3-L1 cells. Int J Mol Med 45: 589-596, 2020.

33. Curcio CA, Johnson M, Rudolf M and Huang JD: The oil spill in ageing Bruch membrane. Br J Ophthalmol 95: 1638-1645, 2011.

34. Liu HY, Zhang YY, Zhu BL, Feng FZ, Yan H, Zhang HY and Zhou B: miR-21 regulates the proliferation and apoptosis of ovarian cancer cells through PTEN/PI3K/AKT. Eur Rev Med Pharmacol Sci 23: 4149-4155, 2019.

(i) $\odot$ This work is licensed under a Creative Commons Attribution-NonCommercial-NoDerivatives 4.0 International (CC BY-NC-ND 4.0) License. 